\title{
Invasive Validation of the Left Ventricular Global Longitudinal Strain for Estimating LV Filling Pressure
}

\author{
Turkan Tan ${ }^{1}$, irem Muge Akbulut ${ }^{2}$, Ayse Demirtola ${ }^{1}$, Nazli Turan Serifler ${ }^{1}$, Nil Ozyuncu ${ }^{1}$, \\ Kutay Vurgun ${ }^{1}$, Kerim Esenboga ${ }^{2}$, Haci Kurklu ${ }^{3}$, Volkan Kozluca ${ }^{4}$, Demet Gerede ${ }^{4}$, Aydan \\ Ongun Ozdemir ${ }^{5}$, Eralp Tutar ${ }^{1}$, and Irem Dincer ${ }^{5}$ \\ ${ }^{1}$ Ankara University Department of Cardiology \\ ${ }^{2}$ Ankara University Faculty of Medicine \\ ${ }^{3}$ Lokman Hekim Universitesi \\ ${ }^{4}$ Ankara University \\ ${ }^{5}$ Ankara University School of Medicine
}

January 20, 2021

\begin{abstract}
Purpose: An elevated left ventricular (LV) filling pressure is the main finding in patients with heart failure with preserved ejection fraction, which is estimated with an algorithm using echocardiographic parameters recommended by the recent American Society of Echocardiography (ASE)/European Association of Cardiovascular Imaging (EACVI) guideline. In this study, we sought to determine the efficacy of LV global longitudinal strain (GLS) to estimate the elevated LV filling pressure. Methods and Results: 73 prospectively selected patients undergoing left ventricular catheterization (mean age 63.19 $\pm 9.64,68.5 \%$ male) participated in this study. Using the algorithm, the LV filling pressure was estimated with the echo parameters obtained within $24 \mathrm{hrs}$ before the catheterization. The LV GLS was measured using the automated functional imaging system (GE, Vivid E9 USA). Invasive LV pre-A pressure corresponding to mean left atrial pressure (LAP) was used as a reference, and $>12 \mathrm{~mm} \mathrm{Hg}$ was defined as elevated. The invasive LV filling pressure was elevated in 43 (58.9\%) and normal in 30 patients (41.1\%). In 9 (12.3\%) patients of 73 are defined as indeterminate based on the 2016 algorithm. Using the ROC method, - $18.1 \%$ of LV GLS estimated the LV filling pressure ( $\mathrm{AUC}=0.79,73 \%$ specificity, $84 \%$ sensitivity) with higher sensitivity compared with the algorithm (AUC=0.76, $77 \%$ specificity, $72 \%$ sensitivity). Conclusions: We confirmed that the LV GLS is feasible and reproducible in estimating LV filling pressure. In addition, LV GLS highly predicts elevated LAP as E/e' and TR jet velocity and may be used as major criteria for the diagnosis of $\mathrm{HFpEF}$
\end{abstract}

\section{Invasive Validation of the Left Ventricular Global Longitudinal Strain for Estimating LV Filling Pressure}

Turkan Seda Tan, $\mathrm{MD}^{1}$; Irem Muge Akbulut, $\mathrm{MD}^{1}$; Ayse Irem Demirtola, $\mathrm{MD}^{1}$; Nazli Turan Serifler, $\mathrm{MD}^{1}$; Nil Ozyuncu, $\mathrm{MD}^{1}$; Veysel Kutay Vurgun, $\mathrm{MD}^{1}$; Kerim Esenboga, $\mathrm{MD}^{1}$; Haci Ali Kurklu, Volkan Kozluca, $\mathrm{MD}^{1}$; $\mathrm{MD}^{2}$, Demet Menekse Gerede Uludag, $\mathrm{MD}^{1}$; Aydan Ongun, $\mathrm{MD}^{1}$; Eralp Tutar, $\mathrm{MD}^{1}$; Irem Dincer, $\mathrm{MD}^{1}$.

${ }^{1}$ Department of Cardiovascular Medicine, Ankara University School of Medicine, Ankara, Turkey

${ }^{2}$ Department of Cardiovascular Medicine, Lokman Hekim University School of Medicine Akay Hospital, Ankara, Turkey

Running title: Estimates of LV filling pressure-LV GLS 
Abstract word count: 248

Manuscript word count: 2788/3523 (including references and figure legends)

\section{Author Declaration}

The authors have no relevant relationships with industry to disclose. No funding was received for this work. We further confirm that any aspect of the work covered in this manuscript that has involved human patients has been conducted with the ethical approval of all relevant bodies and that such approvals are acknowledged within the manuscript. We confirm that the manuscript has been read and approved by all named authors.

Address for correspondence: Turkan Seda Tan, MD; Department of Cardiovascular Medicine, Ankara University School of Medicine, Cebeci Kalp Merkezi, Mamak, Ankara, Turkey (tsedatan@gmail.com; Phone: +90 312-595-6640; Fax: 312-595-6736).

\section{Author e-mail addresses}

Turkan Seda Tan, MD; Department of Cardiovascular Medicine, Ankara University School of Medicine, Ankara, TURKEY

tsedatan@gmail.com ORCID ID: 0000-0002-9349-3371

Irem Muge Akbulut, MD; Department of Cardiovascular Medicine, Ankara University School of Medicine, Ankara, TURKEY

iremuge@yahoo.com

Ayse Irem Demirtola, MD; Department of Cardiovascular Medicine, Ankara University School of Medicine, Ankara, TURKEY

airem90@gmail.com

Nazli Turan Serifler MD; Department of Cardiovascular Medicine, Ankara University School of Medicine, Ankara, TURKEY

nazlituran113@gmail.com

Nil Ozyuncu, MD; Department of Cardiovascular Medicine, Ankara University School of Medicine, Ankara, TURKEY

nilozyuncu@yahoo.com

Veysel Kutay Vurgun, MD; Department of Cardiovascular Medicine, Ankara University School of Medicine, Ankara, TURKEY

kutayvurgun@gmail.com

Kerim Esenboga, MD; Department of Cardiovascular Medicine, Ankara University School of Medicine, Ankara, TURKEY

kerimesenboga@yahoo.com

Haci Ali Kurklu, MD; Department of Cardiovascular Medicine, Lokman Hekim Akay Hospital, Ankara, TURKEY

hacialikurklu@gmail.com

Volkan Kozluca, MD; Department of Cardiovascular Medicine, Ankara University School of Medicine, Ankara, TURKEY

volkankozluca@yahoo.com 
Demet Menekse Gerede Uludag, MD; Department of Cardiovascular Medicine, Ankara University School of Medicine, Ankara, TURKEY

drmeneksegerede@yahoo.com

Aydan Ongun, MD; Department of Cardiovascular Medicine, Ankara University School of Medicine, Ankara, TURKEY

aongun@msn.com

Eralp Tutar, MD; Department of Cardiovascular Medicine, Ankara University School of Medicine, Ankara, TURKEY

eralptutar@gmail.com

Irem Dincer MD; Department of Cardiovascular Medicine, Ankara University School of Medicine, Ankara, TURKEY

iremdincer@yahoo.com

\section{ABSTRACT}

Purpose: An elevated left ventricular (LV) filling pressure is the main finding in patients with heart failure with preserved ejection fraction, which is estimated with an algorithm using echocardiographic parameters recommended by the recent American Society of Echocardiography (ASE)/European Association of Cardiovascular Imaging (EACVI) guideline. In this study, we sought to determine the efficacy of LV global longitudinal strain (GLS) to estimate the elevated LV filling pressure.

Methods and Results : 73 prospectively selected patients undergoing left ventricular catheterization (mean age $63.19 \pm 9.64,68.5 \%$ male) participated in this study. Using the algorithm, the LV filling pressure was estimated with the echo parameters obtained within $24 \mathrm{hrs}$ before the catheterization. The LV GLS was measured using the automated functional imaging system (GE, Vivid E9 USA). Invasive LV pre-A pressure corresponding to mean left atrial pressure (LAP) was used as a reference, and $>12 \mathrm{~mm} \mathrm{Hg}$ was defined as elevated.

The invasive LV filling pressure was elevated in 43 (58.9\%) and normal in 30 patients $(41.1 \%)$. In 9 (12.3\%) patients of 73 are defined as indeterminate based on the 2016 algorithm. Using the ROC method, -18.1\% of LV GLS estimated the LV filling pressure ( $\mathrm{AUC}=0.79,73 \%$ specificity, $84 \%$ sensitivity) with higher sensitivity compared with the algorithm ( $\mathrm{AUC}=0.76,77 \%$ specificity, $72 \%$ sensitivity).

Conclusions : We confirmed that the LV GLS is feasible and reproducible in estimating LV filling pressure. In addition, LV GLS highly predicts elevated LAP as E/e' and TR jet velocity and may be used as major criteria for the diagnosis of $\mathrm{HFpEF}$

Key Words: Diastolic dysfunction, left ventricular filling pressure, heart failure, preserved ejection fraction, echocardiography, left ventricular longitudinal strain

\section{ABBREVATIONS}

HF, Heart failure

LV, Left ventricle

LA, Left atrium

LVEF, Left ventricular ejection fraction

HFpEF, Heart failure with preserved ejection fraction

MI, Myocardial infarction

LBBB, Left bundle branch block 
TR, Tricuspid regurgitation

BNP, Brain natriuretic peptide

\section{INTRODUCTION}

Heart failure is a common public health problem that 6.2 million adults had heart failure (HF) in the United States alone [1]. The description of Heart failure (HF) mainly includes HF symptoms and left ventricular ejection fraction (LVEF), which is established by HF guidelines[2]. HF with preserved ejection fraction (HFpEF) considered as [?]\%50 LV EF[2-5] has complex pathophysiologic mechanisms with challenging diagnostic features[2, 3, 6, 7]. HFpEF prevalence continues to rise due to the aging population, obesity, and some diseases, including diabetes, hypertension, and atrial fibrillation[6, 7]. In fact, up to $50 \%$ of patients in the HF population comprises HFpEF in developed countries.

Patients with HFpEF commonly have normal LV systolic function with a normal systolic and diastolic diameter but have an increased LV wall thickness and left atrial (LA) dilatation. Besides, increased LV filling pressure is an essential finding in patients with HFpEF. Although cardiac catheterization is the gold standard method to demonstrate the elevated LV filling pressure, it is not practical[8]. Therefore, estimating LV filling pressure using transthoracic echocardiography has become a standard method due to its feasibility and reproducibility. Conventional Doppler methods such as diastolic mitral inflow measurement from the tip of mitral leaflets with pulse wave Doppler (PW) and tissue Doppler imaging were previously being used to define diastolic dysfunction [9-12]. To simplify the estimation of LV filling pressure 2016 EACVI/ASE guideline demonstrated a new algorithm [13] using similar echo parameters such as E/e', left atrium volume index(LAVI).

Contrary to general belief, LV systolic function is often impaired and established as one of the main mechanisms of HFpEF with diastolic dysfunction. Regardless of reduced EF, LV systolic dysfunction can be easily identified with LV global longitudinal strain (GLS) method. Furthermore, the Heart Failure Association of the European Society of Cardiology (HFA-ESC) has published a consensus recommendation including a diagnostic algorithm of HFpEF (HFA-PEFF) in 2019, and GLS $<16$ was established as minor functional criteria for the HFA-PEFF algorithm [1]. In this study, we sought to determine the incremental predictive value of LV GLS on the estimation of elevated LV filling pressure in patients with preserved EF

\section{MATERIALS AND METHODS}

Patient Data. This prospective study included 73 adult patients referred clinically indicated left heart catheterization who also had transthoracic echocardiography (TTE) immediately before catheterization. STelevation and non-ST elevation myocardial infarction (MI), EF $<\% 50$, moderate to severe aortic and mitral regurgitation, and moderate to severe aortic and mitral stenosis were excluded. The medical histories, including all clinical and demographic data, were obtained from the electronic medical records. Laboratory results received within $24 \mathrm{hrs}$ before catheterization were obtained. The study protocol was reviewed and approved by the ethical committee.

Transthoracic Echocardiography. Two-dimensional echocardiographic imaging was performed in 73 patients who met the clinical criteria for study inclusion at Ankara University Cardiology Department Twodimensional, color flow, continuous-pulse wave, and tissue Doppler TTE were performed by two experienced physicians using Vivid E9 imaging system (with an M5Sc-D transducer; GE Medical Systems, Chicago, USA) within $24 \mathrm{hrs}$ before left heart catheterization and measurements obtained in a standard manner as recommended by the American Society of Echocardiography. LV dimensions were measured in the parasternal long-axis view at end-systole and end-diastole. LV ejection fraction was calculated from 4 chamber view using the modified Simpson method.

TTE parameters assessed LV diastolic function. Diastolic filling periods, including rapid filling, diastasis, and atrial contraction, were assessed by pulsed wave (PW) Doppler. Mitral inflow at the level of mitral valve leaflet tips was used to measure the peak early (E-wave) and late (A-wave) diastolic flow velocities and calculate the E/A ratio. Besides, tissue Doppler imaging (TDI) using PW was performed with the sample 
volume at the lateral and septal mitral annulus to obtain lateral e' and medial e' velocities. The arithmetic mean of lateral and medial e' were defined as average e', which was used to calculate the E/e' ratio. Peak velocity of the tricuspid regurgitation (TR) jet was measured using continuous-wave Doppler. Left atrial volume was measured using a 4-chamber view and divided body surface area (BSA) to calculate the left atrial volume index (LAVi).

Speckle tracking 2D LV longitudinal strain. Speckle tracking 2D LV longitudinal average and the regional strain was measured using automated functional imaging (AFI). AFI was performed in 73 patients using an E9 imaging system (with a 4V-D transducer; GE Medical Systems, Chicago, USA) and transferred to Echo Pac imaging workstation (Echo Pac imaging system). LV longitudinal strain was performed according to standardized measurements recommended by the 2015 ASE Cardiac Chamber Quantification guideline.

LV catheterization. Left heart catheterization was performed according to the standard procedure by an interventional cardiologist blinded to the echocardiographic data. Invasive LV systolic and diastolic pressure measurements were performed using a 6-Fr pigtail catheter (Boston Scientific, Marlborough, MA) placed in the left ventricle through the femoral or radial artery before the evaluation of coronary artery visualization. The measurements were obtained after the fluid-filled transducer was balanced with the zero level at the mid-axillary line. Continuous pressure tracings were acquired at least three consecutive respiratory cycles. LV pre-A pressure, which corresponds to the mean left atrial pressure(LAP) used as LV filling pressure recommended in the $2016 \mathrm{ASE} / \mathrm{EACVI}$ algorithm, and Pre-A pressure $>12 \mathrm{~mm} \mathrm{Hg}$ confirmed as elevated LV filling pressure.

\section{Statistical Analysis}

Baseline characteristics were presented as mean +-SD for continuous variables and compared using the Student $\mathbf{t}$-test, or percentages for categorical variables differences compared using the chi-square test. A p-value $<0.05$ was defined as statistically significant. Univariate and multivariate analysis based on the logistic regression model were performed to determine the TTE parameters to estimate the elevated LV filling pressure. Only variables with $\mathrm{p}$ value $<0.05$ in univariate analysis were entered into multivariate analysis. Correlation between LV GLS and diastolic parameters were analyzed using the Pearson correlation method. Correlation of invasive LV filling pressure with LV GLS and diastolic parameters were also analyzed using the Pearson correlation method. Sensitivity, specificity, positive predictive value, and negative predictive value of diastolic parameters and LV GLS were analyzed using the Receiver operating characteristic (ROC) analysis based on the Logistic regression method. LV GLS cut-off value was determined by ROC analysis. All data were analyzed using JMP version 14.0 (SAS Institute Inc., Cary, North Carolina)

Inter-observer and intra-observer variability. Images from 10 patients were randomly selected, and a second independent blinded observer measured their images to assesses the inter-observer variability. The first observer who measured all patients' views remeasured the same randomly selected 10 patients' views at least 6 weeks apart from the first measurement. Inter-observer and intra-observer variability were assessed using the Intra Class Correlation Coefficient (ICC) method.

\section{RESULTS}

Baseline characteristics. A total of 73 patients (mean age 63.19+-9.64, 68.5\% male) undergoing left heart catheterization comprised the study population. All patients were divided into two groups by their invasive LV pre-A pressure value. $>12 \mathrm{mmHg}$ group (43 patients; $58.9 \%$; LV pre-A pressure $=17.2+-3.05$ ) was defined as elevated LAP group, [?]12mmHg group (30 patients,41.1\%; LV pre-A pressure=7.5+-2.19) was defined as normal LAP group. Demographic, clinical characteristics, laboratory results, medication use, and TTE results were compared between groups (Table 1). There were no differences in age, gender, medication use, and co-morbidities. Laboratory results (obtained within 24 hrs. prior to LV catheterization), including Hemoglobin, Platelet, ALT, and AST, were also similar between groups. In addition, baseline SBP and SBP during catheterization were not different between groups.

Echocardiographic Measurements. Even though E (E; 0.71+-0.19 vs. 0.62+-0.11, p=0.0093) and E/A ratio 
(E/A; $0.95+-0.32$ vs. $0.73+-0.16, \mathrm{p}=0.0002)$ were significantly higher in patients with elevated LAP group, there were no differences in E/e' ratio and A value between groups. The TR jet velocity was able to measure in 50 patients of 73 patients and significantly higher in the elevated LAP group (TR velocity; $2.75+-0.48$ vs. 2.28+-054, $\mathrm{p}=0.004)$. However, LAVi was similar between groups.

Lv global longitudinal strain. LV longitudinal strain was performed in all patients within 24 hrs. Prior LV catheterization was significantly impaired in patients with elevated LAP group (LV strain; -15.4+-2.83 vs. $-18.9+-2.14, \mathrm{p}<0.0001$ ). Intra-observer (ICC 0.97, CI 95\%:0.91-0.99) and inter-observer (ICC 0.94, CI95\%:0.78-0.98) agreement of strain measurements were excellent.

Univariate and Multivariate Predictors of Elevated LV Filling Pressure: Echocardiographic parameters using the algorithm by 2016 ASE/EACVI guideline and LV GLS were entered the univariate and multivariate logistic regression model to find independent predictors of elevated LV filling pressure. In univariate modeling (Table 2), higher level of E/e' (OR:1.32;95\%CI:1.12-1.55, p<0.0001) and TR jet velocity (OR:1.20;95\%CI:1.04-1.37, $\mathrm{p}=0.002$ ) were highly associated with elevated LV filling pressure. In addition, LV GLS (OR:1.67;95\%CI:1.31-2.14, $\mathrm{p}<0.0001$ ) was univariate predictors of the elevated LAP.

TR jet velocity, E/e', and LV GLS were entered into multivariate analysis (Table 2). After adjusting for E/e' and TR jet velocity, LV GLS (OR:1.76;95\%CI:1.14-2.71, $\mathrm{p}=0.0015)$ was found as an independent predictor of elevated LAP. Furthermore, after adjusting for LV GLS and E/e', TR jet velocity (OR:1.25;95\%CI:1.01$1.55, \mathrm{p}=0.01$ ) was found as an independent predictor for elevated LV filling pressure as well as E/e' (OR:1.45;95\%CI:1.07-1.95, $\mathrm{p}=0.0015)$ was found an independent predictor of elevated LAP pressure after adjusting for LV GLS and TR jet velocity.

Roc Analysis: Estimated LAP was determined using the algorithm recommended by the 2016 ASE/EACVI guideline. In 9(12.3\%) patients of 73 defined as indeterminate based on the algorithm. Of those, 6 patients had elevated Pre-A pressure, and 3 patients had normal pre-A pressure. According to the algorithm, 29 $(39.7 \%)$ patients were defined as elevated LAP, and 35 (48\%) patients were defined as normal LAP. ROC analysis based on the logistic regression model was used to analyze the accuracy of the algorithm to predict the elevated LAP. Also, the individual effect of parameters using the algorithm were analyzed. AUC of LAVi was lower (AUC:0.61, specificity $73.40 \%$, sensitivity $65 \%$ ) to estimate elevated LAP compared with TR velocity (AUC:0.77, specificity $81 \%$, sensitivity $75.80 \%$ ), and E/e'(AUC:0.75, specificity $87 \%$, sensitivity $65 \%$ ) (figure1, Table3). Furthermore, LV GLS had a higher AUC with higher sensitivity (AUC=0.83, specificity $73.4 \%$, sensitivity $86 \%$ ) to estimate elevated LAP compared to echocardiographic parameters using the algorithm. LV longitudinal strain cut-off value was found $-18.1 \%$ based on the ROC curve, and $>-18.1 \%$ of GLS was defined as elevated LAP. GLS -18.1\% had higher sensitivity to predict LAP (AUC:0.79, specificity $73 \%$, sensitivity $84 \%$ ) compared with the algorithm (AUC:0.76, specificity $77 \%$, sensitivity $72 \%$ ) (figure2).

Correlation Analysis: Pearson correlation method was used to assess the correlation between pre-A pressure and echo parameters (Table4). There was no correlation between pre-A and $\mathrm{E}(\mathrm{r}=0.18)$, A $(-0.07)$, and $\operatorname{LAVi}(\mathrm{r}=0.19)$. There was also a weak correlation between invasive Pre-A pressure, $\mathrm{E} / \mathrm{A}(\mathrm{r}=0.27)$, $\mathrm{E} / \mathrm{e}^{\prime}(\mathrm{r}=0.36)$, and TR velocity $(\mathrm{r}=0.36)$. However, there was a moderate correlation between LV GLS $(\mathrm{r}=0.47)$ and invasive pre-A pressure. On the other hand, there was not a good correlation between LV GLS and diastolic echo parameters. $\mathrm{E} / \mathrm{e}^{\prime}(\mathrm{r}=0.34) \mathrm{LAVi}(\mathrm{r}=0.04)$ and $\mathrm{TR}$ velocity $(\mathrm{r}=0.11)$ (figure3).

\section{DISCUSSION}

In our study, we aim to demonstrate the invasive validation of LV GLS in estimating elevated LV filling pressure. We confirmed that the LV GLS highly predicts the elevated LAP in patients with preserved EF such as E/e' and TR jet velocity. We also demonstrated that $-18.1 \%$ of LV GLS had higher sensitivity to estimate LV filling pressure compared with the 2016 ASE/ EACVI algorithm.

As it is known, increased myocardial stiffness and prolongation of active myocardial relaxation are the main reasons for HFpEF, which leads to elevated LV filling pressure. Thus, invasive evaluation of elevated LV filling pressure is the gold standard method to define diastolic dysfunction in patients with HF symptoms. However, 
invasive assessment is not practical and reproducible for all patients with HF symptoms. For this reason, the 2009 American Society of Echocardiography (ASE) and the European Association of Echocardiography (now European Association of Cardiovascular Imaging [EACVI]) guideline simplified and developed a practical algorithm in 2016 guideline to estimate LV filling pressure. However, the studies designed to validate the 2016 ASE/EACVI algorithm with invasive LV filling pressure provided conflicting results. Some of them demonstrated good agreement with invasive LV pressure[2, 3].

Furthermore, the Euro-Filling study demonstrated a substantial sensitivity to diagnose elevated LV filling pressures with the 2016 recommendations in patients undergoing invasive LV end-diastolic pressure measurement. However, they concluded that the algorithm was suboptimal in patients with preserved ejection fraction [4]. On the other contrary, Obokata et al. reported that the new algorithm was specific but poorly sensitive, being able to identify only $34 \%$ of individuals with HFpEF diagnosis [5]. Our study also presented that the new algorithm had good specificity but lower sensitivity to predict LV filling pressure.

Even though transthoracic echocardiography is practical and reproducible to determine the diastolic dysfunction, it is not feasible in some instances, including atrial fibrillation, mitral annular calcification, and indeterminate group defined in the guideline. Almedia et al. showed the increase of indeterminate cases using the 2016 algorithm compared with the 2009 guideline[6]. The inclusion of TR velocity to the new algorithm might be an essential reason for increased indeterminate cases. TR velocity generally reflects severe HFpEF; therefore, the early stage of disease may not be evaluated. Moreover, $30 \%$ of patients show normal resting diastolic function by standard echocardiographic assessment $[7,8]$. Although the parameters' cumulative effect using the algorithm gives substantial information about LV filling pressure, individual parameters have some limitations. Especially E/e' is load-dependent and might affect from angle intonation also have poor predictivity to detect the elevation of LV filling pressures with $37 \%$ estimation [9].

Nevertheless, LAVi is an adequate parameter to estimate the cumulative effect of increased LV filling pressures [10-12]. It might be inadequate to detect early LV diastolic dysfunction since this volumetric parameter reflects essentially the chronic effect of elevated LV filling pressure[13]. Our study observed a week correlation between TR jet velocity, E/e', and invasive pre-A pressure and no correlation between LAVi and invasive pre-A pressure. Additionally, LAVi had lower sensitivity (specificity $73.40 \%$, sensitivity $65 \%$ ) compared with $\mathrm{E} / \mathrm{e}^{\prime}$ and TR jet velocity.

Left ventricular Speckle-tracking global longitudinal strain is a valuable parameter to assess global and regional left ventricular systolic dysfunction. Moreover, LV GLS reflects the longitudinally arranged subendocardial fibers function that is influenced early in disease pathogenesis, allowing detection of even subtle impairment; in contrast, $\mathrm{EF}$ only detects overt systolic failure [14]. It was believed that diastolic impairment of $\mathrm{LV}$ is the main mechanism of $\mathrm{HFpEF}[15,16]$. However, pathophysiological features of HFpEF, including myocardial fibrosis and microvascular dysfunction, can impair both diastolic and systolic function. There is now clear evidence of significant systolic impairment in patients with HFpEF, such as decreased contractility, which is associated with greater mortality [17].

Furthermore, PARAMOUNT study has demonstrated an independent association between NT-proBNP levels and LV GLS and impaired LV GLS as highly predicted adverse outcomes[18, 19]. The 2016 ASE/ESC guideline recommended assessing LV GLS for patients with atrial fibrillation and severe mitral annular calcification. They also recommended LV GLS to provide the discriminative diagnostic capacity in indeterminate groups[12]. Biering-Sorensen, et al. reported that LV GLS for noninvasive evaluation of LV filling pressure acquired good correlation with PCWP both with rest and exertion[20]. Besides, a cut point of $<16 \%$ for LV GLS was included in the HFA-PEFF algorithm as minor criteria for diagnosing HFpEF recommended by the Heart Failure Association of the ESC in 2019 [1]. Considering all of these, we investigated whether the LV GLS is more sensitive in predicting elevated LV filling pressure. We showed that LV GLS had better sensitivity than the 2016 echocardiography algorithm for estimating LV filling pressure. We thought that LV GLS might be added echocardiography algorithm to improve the estimation of LAP pressure. Moreover, deficiency of algorithm, including indeterminate group, atrial fibrillation, and mitral annulus calcification might be evaluated with LV GLS. We also believed that LV GLS may be used as major echocardiographic 
criteria for the HFA-PEFF algorithm and may add incremental value on HFpEF diagnosis.

\section{STUDY LIMITATIONS}

Our study has several limitations with a single center and small patient group. Because of our small amount of patients, the study had only 9 indeterminate patients, so we could not demonstrate whether LV GLS is adequate to define diastolic dysfunction in those patient groups. Besides, all standard echocardiographic measurements and LV GLS were performed in resting, so we could not assess the relationship between LV GLS and impaired functional capacity, which is the highly essential hallmark for HFpEF. Future studies with a multicenter design and a larger study population will be essential to demonstrate the additional effects of GLS on the 2016 ASE/EACVI algorithm as well as on the HFA-PEFF algorithm

\section{CONCLUSION}

Early assessment of systolic impairment is highly crucial in patients with HFpEF due to an independent risk factor for hospitalization and mortality. Our findings suggest that LV GLS might provide an impressive contribution to the conventional algorithm to estimate LAP and may be used not only for diagnostic criteria of HFpEF but also for an early decision of proper treatment

\section{REFERENCES}

1. Pieske, B., et al., How to diagnose heart failure with preserved ejection fraction: the HFA-PEFF diagnostic algorithm: a consensus recommendation from the Heart Failure Association (HFA) of the European Society of Cardiology (ESC). European Heart Journal, 2019. 40 (40): p. 3297-3317.

2. Balaney, B., et al., Invasive Validation of the Echocardiographic Assessment of Left Ventricular Filling Pressures Using the 2016 Diastolic Guidelines: Head-to-Head Comparison with the 2009 Guidelines. J Am Soc Echocardiogr, 2018.31 (1): p. 79-88.

3. Andersen, O.S., et al.,Estimating Left Ventricular Filling Pressure by Echocardiography.Journal of the American College of Cardiology, 2017. 69 (15): p. 1937-1948.

4. Lancellotti, P., et al.,Echo-Doppler estimation of left ventricular filling pressure: results of the multicentre EACVI Euro-Filling study. Eur Heart J Cardiovasc Imaging, 2017. 18 (9): p. 961-968.

5. Obokata, M., et al., Role of Diastolic Stress Testing in the Evaluation for Heart Failure With Preserved Ejection Fraction: A Simultaneous Invasive-Echocardiographic Study. Circulation, 2017. 135 (9): p. 825838.

6. Almeida, J.G., et al., Impact of the 2016 ASE/EACVI recommendations on the prevalence of diastolic dysfunction in the general population. European Heart Journal Cardiovascular Imaging, 2018. 19 (4): p. 380-386.

7. Zile, M.R., et al.,Prevalence and significance of alterations in cardiac structure and function in patients with heart failure and a preserved ejection fraction. Circulation, 2011. 124 (23): p. 2491-501.

8. Obokata, M., Y.N.V. Reddy, and B.A. Borlaug, The Role of Echocardiography in Heart Failure with Preserved Ejection Fraction: What Do We Want from Imaging? Heart Fail Clin, 2019. 15 (2): p. 241-256.

9. Sharifov, O.F., et al.,Diagnostic Accuracy of Tissue Doppler Index E/e' for Evaluating Left Ventricular Filling Pressure and Diastolic Dysfunction/Heart Failure With Preserved Ejection Fraction: A Systematic Review and Meta-Analysis. J Am Heart Assoc, 2016. 5 (1).

10. Morris, D.A., et al.,Potential Usefulness and Clinical Relevance of Adding Left Atrial Strain to Left Atrial Volume Index in the Detection of Left Ventricular Diastolic Dysfunction. JACC Cardiovasc Imaging, 2018. 11 (10): p. 1405-1415.

11. Nagueh, S.F., et al.,Recommendations for the Evaluation of Left Ventricular Diastolic Function by Echocardiography. Journal of the American Society of Echocardiography, 2009. 22 (2): p. 107-133. 
12. Nagueh, S.F., et al.,Recommendations for the Evaluation of Left Ventricular Diastolic Function by Echocardiography: An Update from the American Society of Echocardiography and the European Association of Cardiovascular Imaging. Journal of the American Society of Echocardiography, 2016.29 (4): p. 277-314.

13. Mandoli, G.E., et al., Left atrial strain in left ventricular diastolic dysfunction: have we finally found the missing piece of the puzzle? Heart Fail Rev, 2019.

14. Sengupta, P.P. and J. Narula,Reclassifying heart failure: predominantly subendocardial, subepicardial, and transmural. Heart Fail Clin, 2008. 4 (3): p. 379-82.

15. Borlaug, B.A., et al.,Global cardiovascular reserve dysfunction in heart failure with preserved ejection fraction. J Am Coll Cardiol, 2010. 56 (11): p. 845-54.

16. DeVore, A.D., et al.,Impaired left ventricular global longitudinal strain in patients with heart failure with preserved ejection fraction: insights from the RELAX trial. Eur J Heart Fail, 2017. 19 (7): p. 893-900.

17. Telles, F. and T.H. Marwick,Imaging and Management of Heart Failure and Preserved Ejection Fraction. Curr Treat Options Cardiovasc Med, 2018. 20 (11): p. 90.

18. Solomon, S.D., et al., The angiotensin receptor neprilysin inhibitor LCZ696 in heart failure with preserved ejection fraction: a phase 2 double-blind randomised controlled trial. Lancet, 2012. 380 (9851): p. 1387-95.

19. Kraigher-Krainer, E., et al.,Impaired Systolic Function by Strain Imaging in Heart Failure With Preserved Ejection Fraction. Journal of the American College of Cardiology, 2014. 63 (5): p. 447-456.

20. Biering-Sorensen, T., et al.,Left ventricular deformation at rest predicts exercise-induced elevation in pulmonary artery wedge pressure in patients with unexplained dyspnoea. Eur J Heart Fail, 2017. 19 (1): p. 101-110.

\section{Figure Legends}

Figure1. Receiver-operating curves of estimated LV filling pressure. E/e' AUC $=0.75$ (95\%CI:0.630.85), LAVi AUC $=0.61(95 \%$ CI:0.50-0.73), LV GLS AUC $=0.83(95 \%$ CI:0.71-0.90) and TR jet velocity $\mathrm{AUC}=0.77(95 \% \mathrm{CI}: 0.59-0.87)$,

Figure2. Receiver-operating curves of estimated LV filling pressure. Cut point $-18.1 \%$ of LV GLS $\mathrm{AUC}=0.79(\mathrm{CI} 95 \%: 0.67-0.86, \mathrm{p}<0.0001)$ and the $2016 \mathrm{ASE} / \mathrm{EACVI}$ algorithm AUC=0.76(CI 95\%:0.64-0.84, $\mathrm{p}<0.0001)$ alone.

Figure3. Correlation of Left ventricular longitudinal strain with E/e', LAVi and TR velocity. Weak correlation between left ventricular global longitudinal strain and $\mathrm{E} / \mathrm{e}^{\prime}(\mathrm{r}=0.34, \mathrm{p}=0.0029)$ (A). No correlation between left ventricular global longitudinal strain and LAVi $(\mathrm{r}=0.04, \mathrm{p}=0.68)(\mathrm{B})$. No correlation between LV global longitudinal strain and TR velocity $(\mathrm{C})$.

Figure4. An example of a study patients' invasive LV filling pressure and LV GLS results. Impaired LV GLS (-15.2\%) matched the invasive Pre-A pressure, which was elevated. 

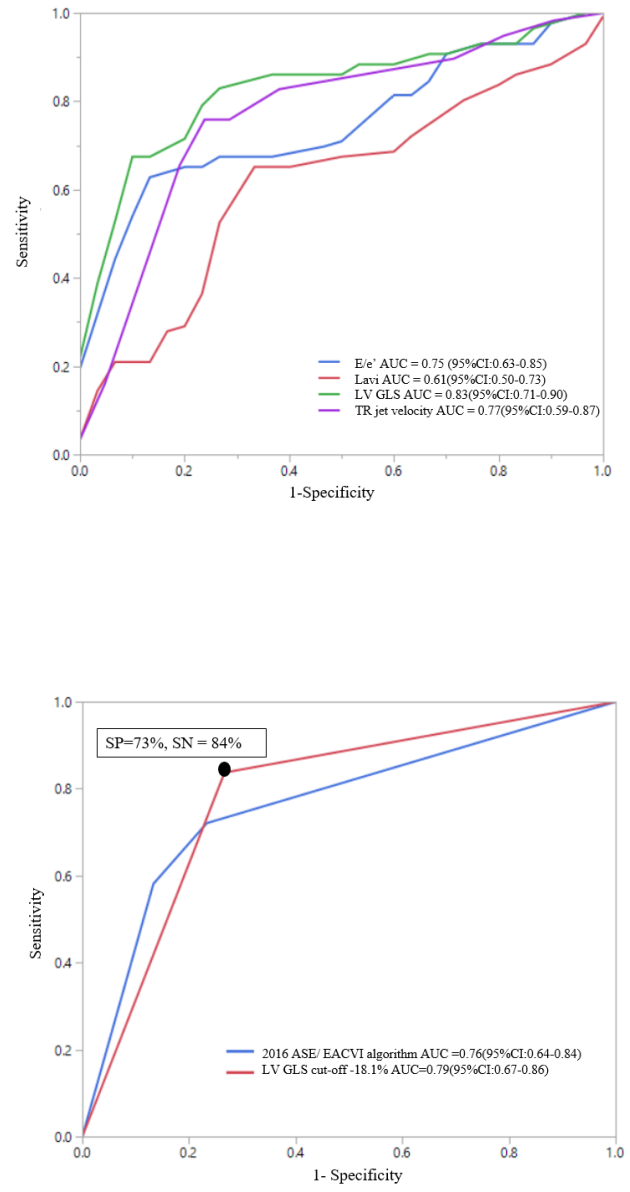

(4) (b) (2) (영) (1)

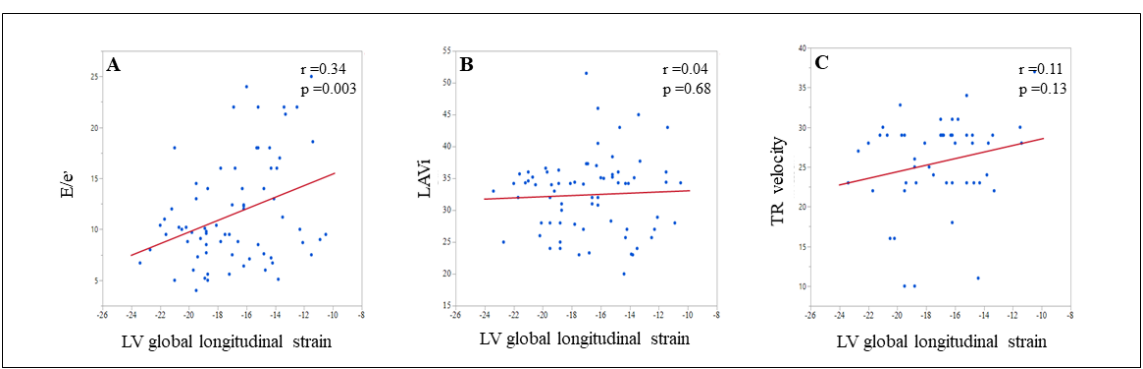




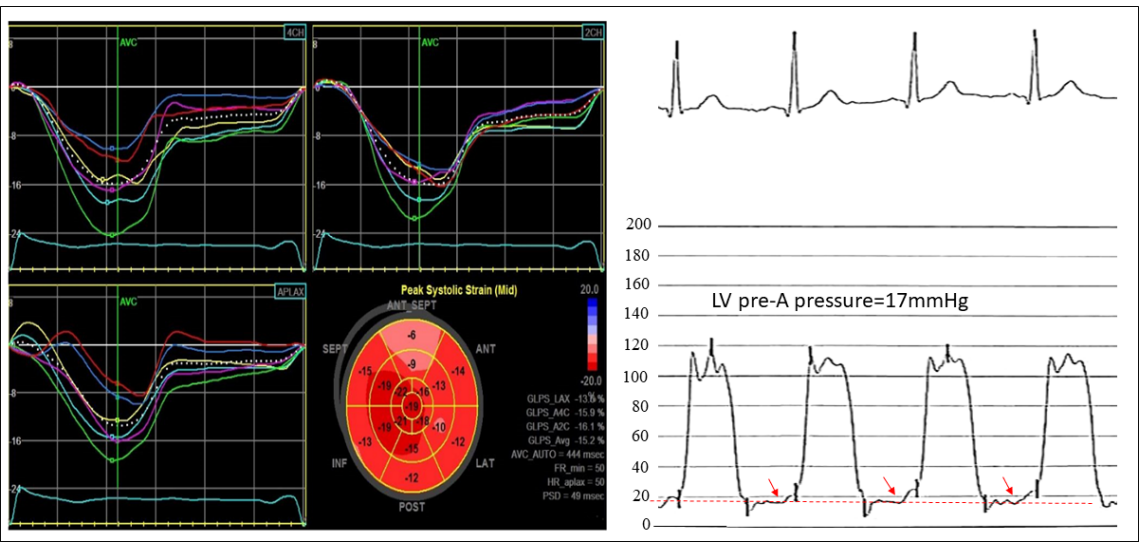

